\title{
Phase Transition between Synchronous and Asynchronous Updating Algorithms
}

\author{
Filippo Radicchi • Daniele Vilone • \\ Hildegard Meyer-Ortmanns
}

Received: 11 May 2007 / Accepted: 27 August 2007 / Published online: 21 September 2007

(C) Springer Science+Business Media, LLC 2007

\begin{abstract}
We update a one-dimensional chain of Ising spins of length $L$ with algorithms which are parameterized by the probability $p$ for a certain site to get updated in one time step. The result of the update event itself is determined by the energy change due to the local change in the configuration. In this way we interpolate between the Metropolis algorithm at zero temperature when $p$ is of the order of $1 / L$ and $L$ is large, and a synchronous deterministic updating procedure for $p=1$. As a function of $p$ we observe a phase transition between the stationary states to which the algorithm drives the system. These are non-absorbing stationary states with antiferromagnetic domains for $p>p_{c}$, and absorbing states with ferromagnetic domains for $p \leq p_{c}$. This means that above this transition the stationary states have lost any remnants of the ferromagnetic Ising interaction. A measurement of the critical exponents shows that this transition belongs to the universality class of parity conservation.
\end{abstract}

Keywords Nonequilibrium and irreversible dynamics · Phase transitions

\section{Introduction}

The issue of synchronous versus asynchronous updating algorithms has attracted much attention in connection with Boolean networks [1, 2], neural networks [3, 4], biological networks $[5,6]$ and game theory $[7,8]$. In a synchronous updating scheme all the units of the system are updated at the same time. Asynchronous updating means that not all units are updated at the same time. The algorithm can be asynchronous in the sense that each unit is updated according to its own clock, as in distributed systems for parallel processing [9], or it is asynchronous in the sense that only one randomly chosen unit is updated at each step,

F. Radicchi $(\bowtie) \cdot$ D. Vilone $\cdot$ H. Meyer-Ortmanns

School of Engineering and Science, Jacobs University Bremen, P.O. Box 75056, 28725 Bremen,

Germany

e-mail: f.radicchi@gmail.com

H. Meyer-Ortmanns

e-mail: h.ortmanns@jacobs-university.de 
as in Monte Carlo algorithms [10]. In the context of thermal equilibrium dynamics, updating algorithms like the Metropolis algorithm [11] are designed in a way that they drive the configurations to a set that is representative for the Boltzmann equilibrium distribution. The dynamics of the algorithm then enters only in an intermediate step, it is not representative for the intrinsic equilibrium dynamics of the system that is determined by the Hamiltonian. In out-of-equilibrium systems the updating scheme plays a more prominent role. The number of attractors in Boolean networks, for example, increases exponentially with the system size [12] for synchronous update, and with a power for critical Boolean networks [13] for asynchronous update. The phase diagrams of the Hopfield neural network model [3, 14] and the Blume-Emery-Griffiths model [15] depend on the updating mode as well, while those of the Q-state Ising model [16, 17], and the Sherrington-Kirkpatrick spin glass [18] are independent on the used scheme.

Probably neither a completely synchronous nor a random asynchronous update is realistic for natural systems. Here we interpolate between these two extreme cases not in a more realistic way, but in a way that allows to identify a phase transition between the stationary states. Synchronous updates may appear as non-physical updates for an Ising model as we consider later, since they do not lead to an equilibrium at minimal energy. Our main goal here is not to propose the synchronous update as another physically relevant mode, but to illustrate the sensitive dependence of the stationary states on the updating scheme even in case of a simple model like a chain of Ising spins. In the context of biological networks such as the yeast cell cycle [19], it depends in general on the concrete dynamics and the function of the network whether the biological "updating" has to proceed in a well ordered deterministic way, or whether it can proceed more or less randomly. Therefore it seems to us not to be meaningful to call synchronous updates the non-physical and asynchronous the physical modes, it depends on the actual case considered. As we shall show, our interpolation by means of a single parameter $p$ is equivalent to the cellular automaton version of the non-equilibrium kinetic Ising model (NEKIMCA) introduced in [20] and further considered in [21]. The equivalence refers to the elementary processes in terms of diffusion, annihilation and even-branching processes that preserve parity. Therefore the phase transition between the stationary states should belong to the parity conservation universality class. This is the universality class shared also by the models of branching and annihilation random walk with an even number of offspring [22], the non-equilibrium kinetic Ising model (NEKIM) [20, 23] and the cellular automaton version NEKIMCA [20]. Our analysis of critical exponents confirms this expectation.

\section{The Dynamics}

We consider a one-dimensional lattice of length $L$. To each site $i, i=1, \ldots, L$, of this chain we assign a spin variable $\sigma_{i}$, where $\sigma_{i}$ takes the values +1 or -1 . The Hamiltonian of the system is given by $H=-J \sum_{i=1}^{L} \sigma_{i} \sigma_{i+1}$, where $J$ is the coupling constant between neighboring sites. Here we consider ferromagnetic couplings so that $J=1$. Moreover, for definiteness we choose periodic boundary conditions so that $\sigma_{L+1}=\sigma_{1}$. The results will not depend on this choice.

A canonical local stochastic updating algorithm for studying thermodynamic equilibrium features of the Ising model is the Metropolis algorithm [11]. Given a configuration of the system $\Sigma(t)=\left\{\sigma_{i}(t)\right\}$ at time $t$, we pick up one site $j$ at random and flip its spin with probability $P_{j}(t)=\min \left\{1, \exp \left[-\beta \Delta E_{j}(t)\right]\right\}$. Here $\beta=1 / k T$, with $k$ the Boltzmann constant and $T$ the temperature, while $\Delta E_{j}(t)=2 \sigma_{j}(t)\left[\sigma_{j-1}(t)+\sigma_{j+1}(t)\right]$ is the difference in local 
energy that a flip of $\sigma_{j}$ would induce. In particular, for zero temperature an increase in energy of the resulting configuration is always rejected. After the single update of the $j$ th site the time increases by $t \rightarrow t+1 / L=t^{\prime}$ (and $L$ single updates are considered as one time unit). The new configuration is given by $\Sigma\left(t^{\prime}\right)=\left\{\sigma_{i}(t) ; \sigma_{j}\left(t^{\prime}\right)\right\}$, where all sites $i \neq j$ have the same spin value as they have at time $t$ and only the spin of the $j$ th site may be flipped. In this context we emphasize that the Metropolis algorithm is fully asynchronous in the sense that we have at most one spin flip per single update event.

Here we are no longer interested in the equilibrium properties of the Ising model, but in the algorithmic dynamics itself when it is applied to Ising spins. Therefore we give up the asynchrony of the algorithm. Given the configuration $\Sigma(t)=\left\{\sigma_{i}(t)\right\}$ at time $t$, we visit all sites and select each of them with probability $p$ as a candidate to get flipped. The selected sites are $j_{1}, \ldots, j_{m}$ and $m$, the total number of selected sites, is a random integer obeying the binomial distribution $B(m, L, p)=\left(\begin{array}{l}L \\ m\end{array}\right) p^{m}(1-p)^{L-m}$. Each of the $m$ selected sites is then updated according to the Metropolis rule at temperature zero, so that the spin of the $j_{v}$ th site is flipped with probability $P_{j_{v}}(t), \forall v=1, \ldots, m$. After one step of the algorithm, the time increases as $t \rightarrow t+p=t^{\prime}$, and the new configuration is $\Sigma\left(t^{\prime}\right)=\left\{\sigma_{i}(t) ; \sigma_{j_{1}}\left(t^{\prime}\right) ; \ldots ; \sigma_{j_{m}}\left(t^{\prime}\right)\right\}$, where all sites $i \neq j_{1}, \ldots, j_{m}$ have the same spin value as they have at time $t$, while the spins of the $m$ selected sites are at disposal for being synchronously flipped. One time unit has passed when the average number of update events equals to the total number of sites $L$. By varying $p$ we can "tune" the algorithm from asynchrony, for $p$ of the order of $1 / L$, to synchrony, for $p=1$. In particular, for $p$ of the order of $1 / L$ and for sufficiently large values of $L$ we recover the usual Metropolis algorithm, that is, when two or more simultaneous selections, which occur at most with probability of the order of $1 / L^{2}$, become negligible. Differently from the standard Metropolis algorithm at zero temperature, for general values of $p$, the total energy of the new configuration $\Sigma\left(t^{\prime}\right)$ can be increased with respect to the total energy of the old configuration $\Sigma(t)$. Moreover, the large-time configurations $\Sigma(t \rightarrow \infty)$ do no longer obey the Boltzmann distribution. In particular, in the fully synchronous update, all spins (due to $p=1$ ) having a neighboring spin of opposite sign before the synchronous update, will be updated and flipped at the same time so that the global energy of the resulting configuration may increase.

In this paper, for simplicity, we focus on the case of zero temperature. The equilibrium ground-state of the one-dimensional Ising ferromagnet at zero temperature is one of the two ferromagnetic states with all spins positive or negative. In contrast, the completely synchronized dynamics does not drive the system to the ground state, but acts as parallel algorithm and amounts to a deterministic map $T: \Sigma(t+1)=T \Sigma(t)$, for all $t$. After a transient time $t_{0} \leq L / 2$, the algorithm drives the system into a cycle of length two [17], where the system "flips" between two configurations, $\Sigma_{1}=\left\{{ }^{1} \sigma_{i}\right\}$ and $\Sigma_{2}=\left\{{ }^{2} \sigma_{i}\right\}$, such that $\Sigma_{2}=T \Sigma_{1}$ and $\Sigma_{1}=T \Sigma_{2}$, for all $t \geq t_{0}$. In particular, these configurations result from each other by an overall flip of signs in the sense that $\Sigma_{2}=\left\{-{ }^{1} \sigma_{i}\right\}$ and $\Sigma_{1}=\left\{-{ }^{2} \sigma_{i}\right\}$. Therefore, it is natural to study intermediate values of $p$, in particular to focus on the transition between the ferromagnetic and the anti-ferromagnetic configurations of the final state.

Let us consider the active bonds of the system, where we define a bond as active if it connects two sites with opposite spins. As remnant of the zero-temperature Ising model only sites belonging to at least one active bond can flip and do flip if they are selected as candidates for the updating. Only a few elementary processes, that involve active bonds, can take place: diffusion, annihilation and creation in the sense of even-branching processes. To have a clear notation, let us indicate as $\uparrow$ a site with positive spin and as $\downarrow$ a site with negative spin. Consider, for example, a local configuration such as $\cdots \uparrow \uparrow \downarrow \downarrow \cdots$ at time $t$ : at time $t+p$ it can evolve to $\cdots \uparrow \uparrow \uparrow \downarrow \cdots$ or to $\cdots \uparrow \downarrow \downarrow \downarrow \cdots$, depending on whether 
they are selected for an update that happens with probability $2 p(1-p)$ [diffusion or random walk], or it evolves to $\cdots \uparrow \downarrow \uparrow \downarrow \cdots$ with probability $p^{2}$ [branching], or it remains unchanged with probability $(1-p)^{2}$. Using the same rules, a local configuration such as $\cdots \uparrow \uparrow \downarrow \uparrow \uparrow \cdots$ at time $t$, later, at time $t+p$, can become $\cdots \uparrow \downarrow \uparrow \downarrow \uparrow \cdots$ with probability $p^{3}$ [branching], or $\cdots \uparrow \uparrow \uparrow \uparrow \uparrow \cdots$ with probability $p(1-p)^{2}$ [annihilation], etc. These three processes, diffusion (random walk), annihilation and branching, correspond to the three elementary processes in the NEKIM [20,23], that is random walk and annihilation due to a Glauber-spin flip transition rate, and spin exchange with branching due to a Kawasakiexchange rate, and in common with them, they preserve parity of active bonds (called kinks in the NEKIM). Parity corresponds to the number of active bonds modulo 2 . The Glauberspin flip transition rate, as it is used in NEKIM, is a generalization of the spin-flip rate as it was used in the seminal paper by Glauber [24]. Glauber predicted the time evolution of the expectation value of a single spin and spin products at temperature $T$ if the spin-flip rate is parameterized by two parameters, $\gamma$ and $\alpha$; the parameter $\gamma$ determines the tendency of a spin towards alignment with the neighbors (it is one for zero temperature, so that our algorithm corresponds to $\gamma=1$ ), and $\alpha$ sets the time scale on which the transitions take place; in our parametrization this scale is determined by $p$. In addition to the processes in Glauber's dynamics we have branching processes. Their frequency of occurrence depends on the choice of the parameter $p$. For $p$ of the order of $1 / L$ and $T=0$ we obtain a ferromagnetic stationary state in agreement with Glauber's predicted time behavior of the total magnetization for $\gamma=1$, the case in which the magnetization does not decay with time.

\section{Results}

The former considerations suggest that the transition between ferromagnetic and antiferromagnetic behavior (without active bonds and with dominance of active bonds, respectively) is given by the competition of annihilation and branching of active bonds. In particular, the branching is favored by a synchronous updating scheme, because a new couple of active bonds can be created only if two neighboring sites simultaneously flip their spins, so that the flips amount to an exchange of spins. Therefore the transition between stationary states (ferromagnetic and anti-ferromagnetic ones) can be considered as a transition between the asynchronous/synchronous updating schemes. Similarly, in case of the NEKIMCA, that is a stochastic cellular automaton version of the NEKIM, parity-conserving branching of active bonds is generated due to the synchronous update of neighboring sites (without an explicit spin exchange rate as in the mere NEKIM). Favoring active-bond branching due to synchronous updates in the NEKIMCA, also there supports the convergence to an active state as in our case. Figure 1 with active bonds represented as black dots shows a qualitative picture of the phase transition.

We plot the time evolution of an isolated active bond (that is not the only one in the system) for three different values of $p$. We only display the intermediate part of the lattice

Fig. 1 Evolution of an isolated active bond in the subcritical, critical and supercritical regimes. We show only the part around the initial active bond. From left to right: $p=0.39, p=0.41=p_{c}$, $p=0.43$
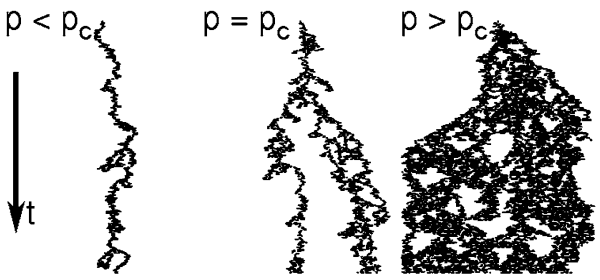
around the initial active bond. The initial configuration is chosen as $\cdots \uparrow \uparrow \downarrow \downarrow \cdots$. In the supercritical regime $p>p_{c}$, the average number of branching is larger than the average number of annihilations, so that the active bonds spread over the entire system. In the critical and subcritical regime $p \leq p_{c}$, annihilation and branching processes are balanced and the active bonds do not spread over the system, but remain confined to a finite region.

\subsection{The Critical Point}

In order to obtain a quantitative description of the transition, we use as order parameter the density of active bonds

$$
\rho=\frac{1}{2 L} \sum_{i=1}^{L} 1-\sigma_{i} \sigma_{i+1},
$$

given by the ratio of the number of active bonds and the total number of bonds in the lattice. The initial condition is chosen as $\rho(0)=1$, so that the lattice is fully occupied with active bonds. All data points are obtained from averaging over at least $10^{3}$ realizations and up to $10^{5}$ realizations for small sizes of the lattice. Here, these values of $L$ are considered as the large-volume limit in the following. Let us first determine the critical probability $p_{c}$.

In Fig. 2(A) we plot the time behavior of $\rho$ for three different values of $p$ and $L=10^{4}$. As we can see, for $p=0.41 \rho(t) \sim t^{-\delta}$ with $\delta=0.286(1)$, while for $p=0.40 \rho(t)$ decreases with negative curvature, for $p=0.42 \rho(t)$ increases with positive curvature. The positive curvature characterizes the different phase. Therefore, within the given accuracy, we locate the critical threshold $p_{c}$ in the large-volume limit as the largest value for which the curvature is non-positive. In this way we obtain $p_{c}=0.41(1)$ as the critical point. In order to determine $p_{c}$ with higher precision we should increase the linear size of the lattice $L$ and the time for observing the positive curvature of $\rho$. Here we do not perform this kind of computationally expensive simulations, but calculate those critical exponents which do not require a higher precision in $p_{c}$. The fact that we observe a phase transition at $p_{c}>0$, corresponding to a finite branching rate $\sigma_{c}>0$, can be understood from the analytic theory of branching and annihilating random walks by Cardy and Täuber [25]. By means of a fieldtheoretic dynamical renormalization group, Cardy and Täuber identified the fluctuations which are responsible for shifting the critical value of the branching rate $\sigma_{c}$ to values larger than zero for dimensions $d<d_{c}^{\prime}=4 / 3$, in particular for $d=1$ and $m$ even as in our case, where $m$ enters the branching according to $A \rightarrow(m+1) A$, and the number of "particles" (active bonds) is locally conserved modulo two. (The measured values of the critical exponents that we consider in the next section cannot be reproduced by this analytic approach.)

\subsection{Critical Exponents}

(a) Critical exponent $\delta \quad$ Along the determination of $p_{c}$ we have already read off the exponent $\delta$ from the time evolution of $\rho$ : for $p \leq p_{c}$ and in the thermodynamic limit $L \rightarrow \infty$, we observed $\rho(t) \sim t^{-\alpha(p)}$, with $\alpha(p)$ a continuous and monotonically decreasing function of $p$ and enclosed by curves with $\alpha(p)=1 / 2$ for $p \rightarrow 0^{+}$, as in the case of the standard Metropolis algorithm $[10,26]$, and $\alpha\left(p_{c}\right)=\delta$, as observed in our numerical simulations. Note that the power-law decay does not only hold at $p_{c}$, but also below $p_{c}$ in the large-volume limit. Therefore the negative curvature in the subcritical regime is actually a finite-size effect and not an effect of $p<p_{c}$. Also in the supercritical regime the curve bends down to zero as a finite-size effect after a sufficiently large time, but the positive curvature signals the onset of the new (supercritical) phase. 
Fig. 2 (A) Time decay of the density of active bonds $\rho$ in the subcritical $(p=0.40)$, critical $\left(p=0.41=p_{c}\right)$ and supercritical $(p=0.42)$ regime, from bottom to top, respectively. (B) Average density of active bonds $\rho_{S}$ surviving up to time $t$ over samples, plotted as a function of time at the critical point for $L=16,32,64,128,256,512$, 1024, 2048, 4096 and 6144, from top to bottom respectively. The dotted line has a slope of $-\delta=-0.286(1)$
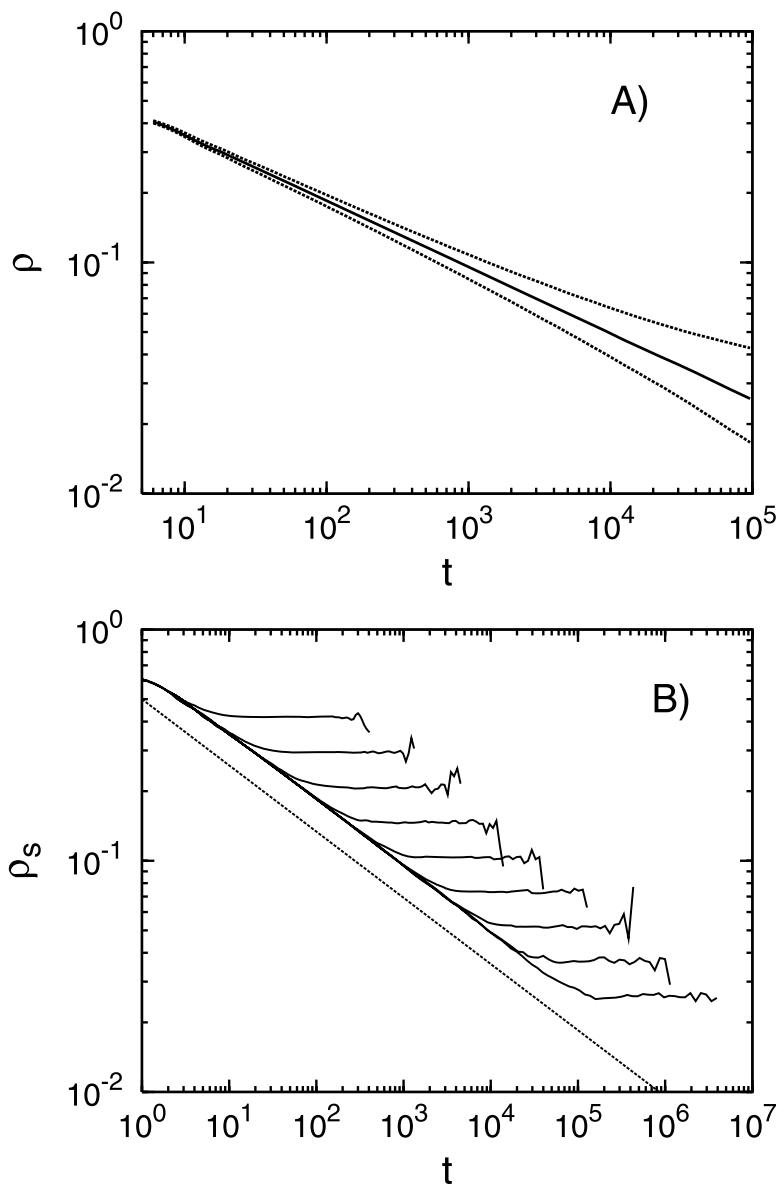

\subsubsection{Finite-Size Scaling Analysis}

Finite-size effects in the density of active bonds are manifest in two ways: if we follow the time evolution of a certain configuration of a chain of length $L$, we observe a powerlaw decay of active bonds up to a certain time $\tau_{d}$ for $p \leq p_{c}$. After that, either the density drops to zero faster than a power-law, this happens for most configurations, for which a fluctuation drives the system into the absorbing state, or, in the minority of evolutions, the number of active bonds fluctuates around a plateau, before the plateau drops to zero in the end at time $\tau_{s}$. Configurations of this minority are called surviving configurations up to time $\tau_{s}$. Now it is easier to locate the onset of the plateau than the onset of a faster decay, and therefore to study the finite-size scaling of the density of active bonds $\rho_{s}$ of surviving configurations as a function of $L$, averaged only over the surviving realizations [22].

(a) Critical exponents $\beta$ and $\nu_{\perp} \quad$ In Fig. 2(B) we plot the time behavior of $\rho_{s}$ at the critical point for several values of the size $L$. As we can see, after an initial transient in which $\rho_{s}$ decreases as $t^{-\delta}$, it reaches a stationary value depending on $L$. This value vanishes in the thermodynamical limit, since the plateau is a finite-size effect. We average along the values of the plateau (since averaging over time and over different realizations are assumed 
Fig. 3 (A) Average density of active sites over the surviving samples $\rho_{S}$ as a function of size $L$ of the lattice for $p=0.40$ (empty dots) 0.41 (full dots) and 0.42 (empty squares). The full line has slope

$-\beta / v_{\perp}=-0.51(1)$. (B) Average relaxation time $\tau$ as a function of the size $L$ of the lattice for $p=0.40,0.41$ and 0.42 (the symbols are the same as in $(A)$ ). The full line has slope $z=v_{\|} / v_{\perp}=1.746(2)$
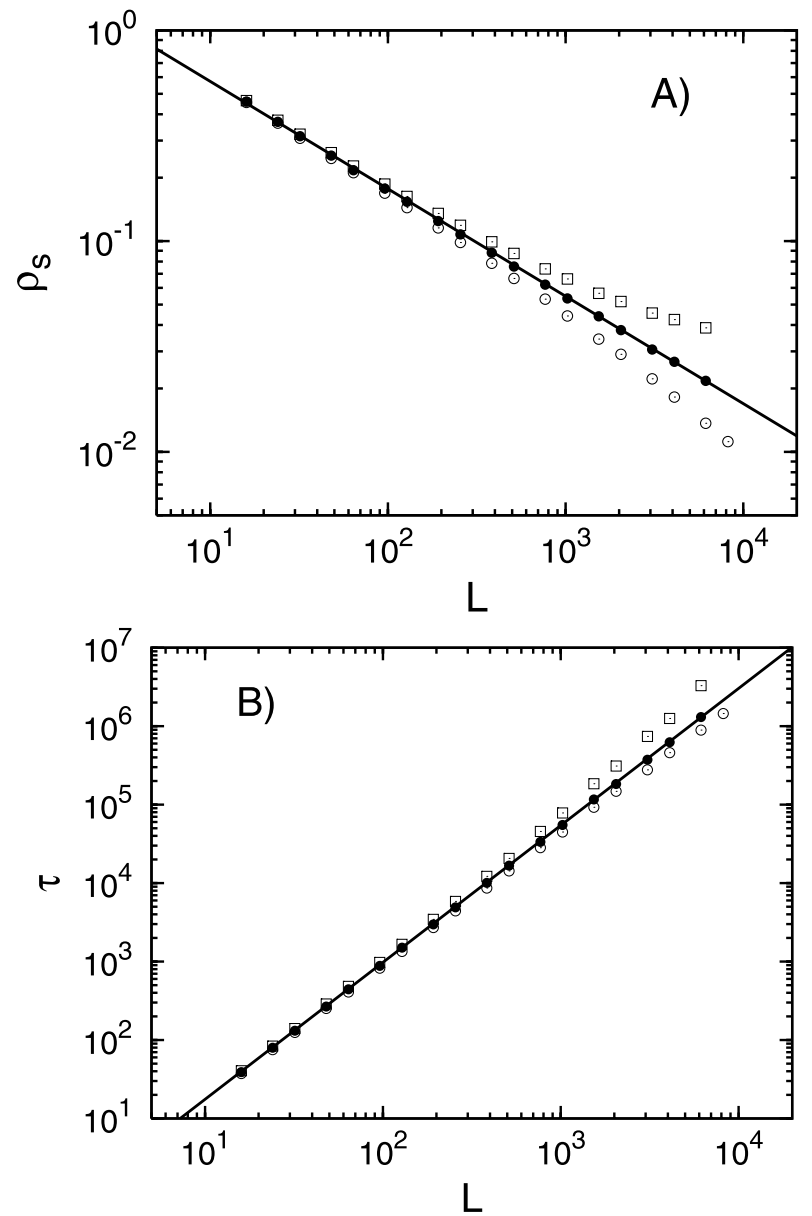

to be equivalent), for different system sizes. In Fig. 3(A) this average value is plotted as a function of $L$ for three values of $p\left(p=0.40, p=0.41=p_{c}\right.$ and $\left.p=0.42\right)$. Again, at the critical point we find a power law decay $\rho_{s} \sim L^{-\beta / \nu_{\perp}}$, with $\beta / \nu_{\perp}=0.51(1)$, while the decay deviates from the power-law behavior in both the subcritical and the supercritical regimes. (Here the exponent $\beta$ characterizes the behavior of the order parameter $\rho(p) \sim\left|p_{c}-p\right|^{\beta}$, $v_{\perp}$ the spatial correlation length $\xi(p) \sim\left|p_{c}-p\right|^{-v_{\perp}}$ both in the vicinity of $p_{c}$.)

(b) Critical exponents $v_{\|}, \eta$ and $\gamma \quad$ Moreover, from the finite-size scaling analysis we calculate the dynamical exponent $z=v_{\|} / v_{\perp}$, where $v_{\|}$characterizes the time-like correlation length $\tau(p) \sim\left|p_{c}-p\right|^{-v_{\|}}$. The exponent $z$ is derived from the relaxation time $\tau$ needed by a finite system to reach the absorbing configuration $[\rho(t \geq \tau)=0]$, starting from a chain fully occupied with active bonds. The unit of the relaxation time is again given by the time that has passed until the average number of update events equals to $L$. In Fig. 3(B) $\tau$ is plotted as a function of $L$ for $p=0.40, p=0.41=p_{c}$ and $p=0.42$. At $p=p_{c}$ we find again a power-law dependence $\tau \sim L^{z}$, with $z=v_{\|} / v_{\perp}=1.746(2)$, while for different values of $p$ $\tau$ behaves differently from a power-law behavior, as it is seen in Fig. 3(B). Furthermore, in 
Fig. 4 The main plot shows the finite-size scaling of the density of active bonds $\rho$ (see (2)). The inset shows the unscaled data, where the dotted line has a slope equal to $-\delta$. The datasets are the same as in Figs. 2 and 3

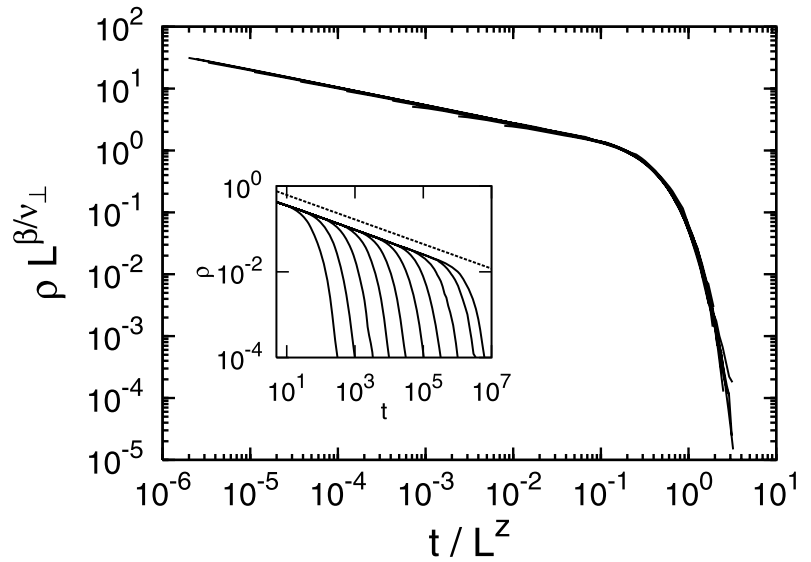

Fig. 5 Susceptibility $\chi_{s}$ as a function of the linear size of the lattice $L . \chi_{s}$ is plotted for $p=0.40,0.41$ and 0.42 , where the symbols adopted are the same as in Fig. 3. As it is shown at the critical point $p_{c}=0.41, \chi_{s}$ is a constant, that means $\gamma \simeq 0$. The datasets are the same as in Figs. 2 and 3

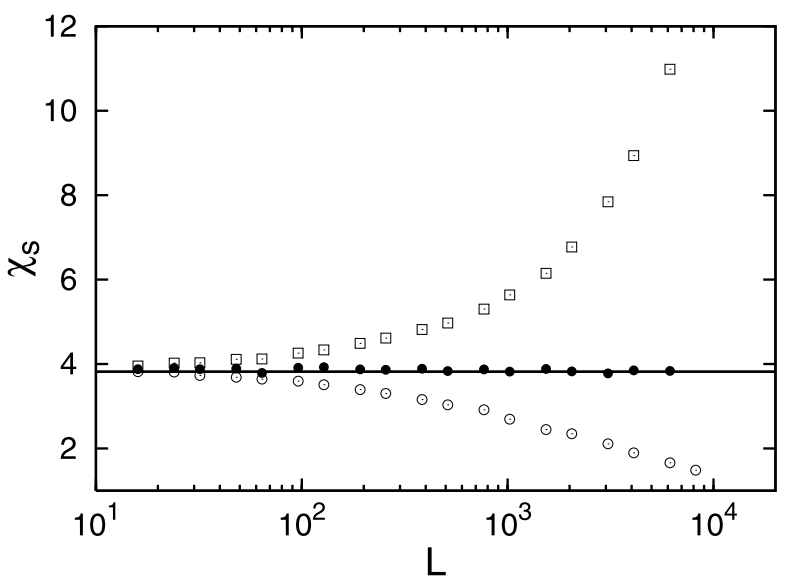

Fig. 4 we verify the finite-size scaling relation at $p_{c}$

$$
\rho(L, t) \sim L^{-\beta / v_{\perp}} f\left(t / L^{z}\right),
$$

where $f(\cdot)$ is a suitable universal function.

Finally, we determine the static exponent $\gamma$ from the growth of fluctuations in the orderparameter susceptibility, defined as $\chi_{s}=L\left(\left\langle\rho_{s}^{2}\right\rangle-\left\langle\rho_{s}\right\rangle^{2}\right.$ ), via $\chi_{s} \sim L^{\gamma / \nu_{\perp}}$ at $p_{c}$ (where the index $s$ again refers to an average over the surviving configurations). We find $\gamma \simeq 0$, as it is shown in Fig. 5.

In the large-volume limit, we further determine the dynamical exponent $\eta$ and check the exponents $\delta$ and $z$ in the following way. Starting from a configuration like $\cdots \uparrow \uparrow \downarrow \uparrow \uparrow \cdots$, that is a ferromagnetic configuration with only two active bonds, we numerically compute the survival probability $P(t)$ (that is the probability that the system had not entered the absorbing state up to time $t$ ), the average number of active bonds $\bar{n}(t)$ and the average mean square distance of spreading $\bar{R}^{2}(t)$ from an arbitrary selected site. These quantities are expected to behave at the critical point $p_{c}$ like $P(t) \sim t^{-\delta}, \bar{n}(t) \sim t^{\eta}$ and $R^{2}(t) \sim t^{2 / z}$ [27], respectively. The critical exponent $\delta$ can be easily calculated by looking at the time behav- 
Fig. 6 Time dependent behavior of the effective exponents $\delta(t)(\mathbf{A}), \eta(t)(\mathbf{B})$ and $2 / z(t)(\mathbf{C})$. We plot these quantities for different values of $p$ : $p_{c}>p=0.4$ (dashed line $)$, $p_{c}=p=0.41$ (bold line) and $p_{c}<p=0.42$ (solid line)
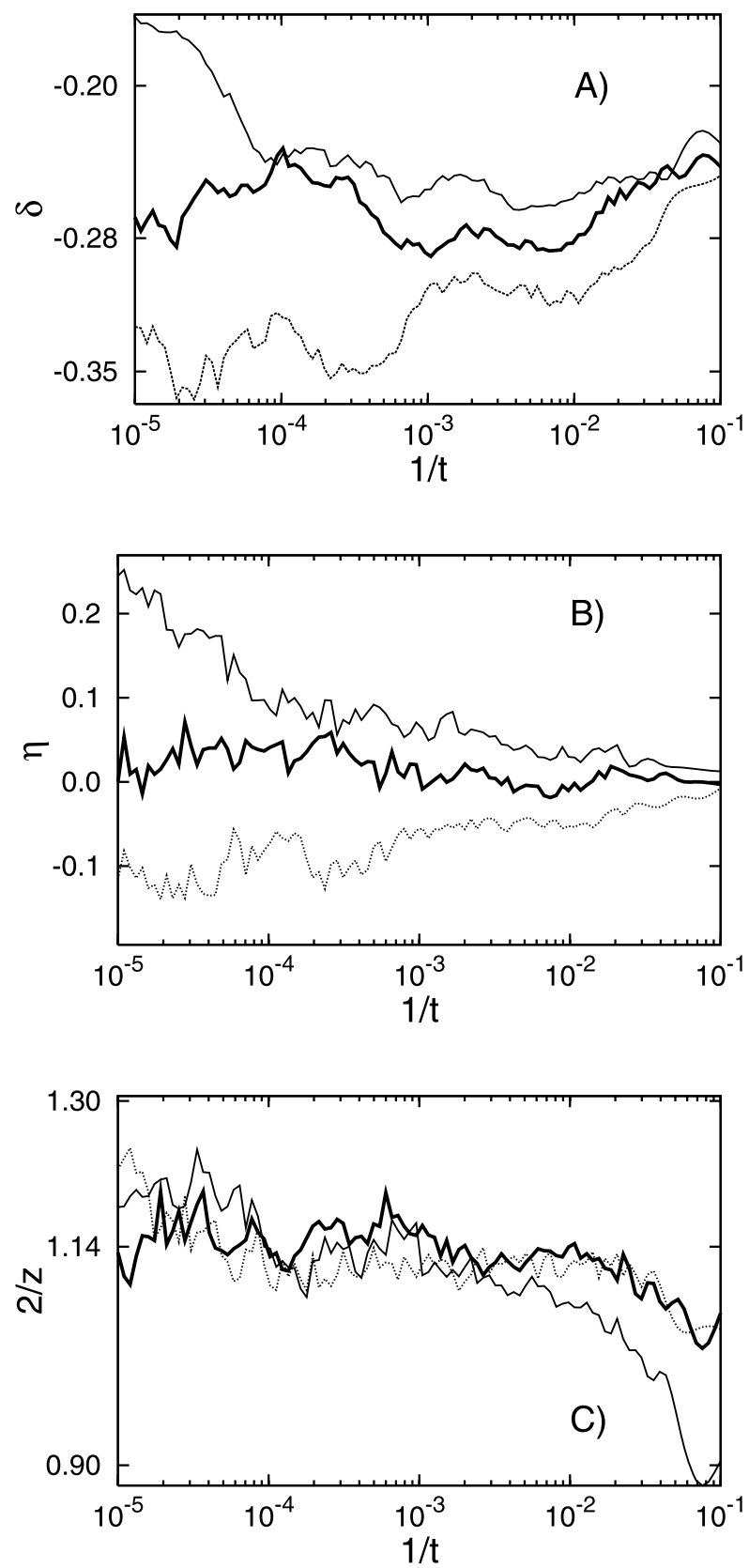

ior of $P(t)$. In Fig. 6(A) we report the effective exponent [27], calculated according to the formula

$$
-\delta(t)=\frac{\log _{10}[P(t) / P(t / b)]}{\log _{10}(b)},
$$


where $b$ is a fit parameter. The right value of the critical exponent $\delta$ is then given by

$$
\delta=\lim _{t \rightarrow \infty} \delta(t) .
$$

In our numerical simulations we consider a lattice of size $L=6144$ and we average over $10^{4}$ realizations. The same simulations and similar definitions for the effective exponents $\eta(t)$ and $2 / z(t)$ lead to the numerical evaluation of $\eta$ (Fig. 6(B)) and $2 / z$ (Fig. 6(C)). From these simulations we find $\eta \simeq 0$ and values of $\delta$ and $z$ consistent with the former ones within the error bars. In particular in Fig. 6 we choose $b=5$ for the evaluation of the effective exponents, but we verified the consistency of our numerical measurements also for other values of $b$.

\section{Conclusions}

In conclusion, the critical exponents we have measured and reported in Table 1 are consistent with the ones conjectured for the parity conservation (PC) universality class [23, 27-32].

This is expected because periodic boundary conditions for Ising spins as well as the updating rules we used preserve the parity of the number of active bonds. Moreover, for free boundary conditions only the boundaries may violate the conservation of parity that leads to a negligible effect. In terms of elementary processes (diffusion, annihilation and branching) the model is equivalent to the branching and annihilation random walk with an even number of offsprings. We use the same elementary processes as those used in NEKIM or NEKIMCA, for which the phase transition between active and absorbing states is known to belong to the PC universality class. In contrast to the NEKIM(CA), our algorithm is parameterized in a different, but equivalent way, for which the frequency of branching processes is tuned via the parameter $p$, that was introduced to directly interpolate between synchronous and asynchronous updates, and not between Glauber and Kawasaki dynamics as in case of the NEKIM. Also the background for both parameterizations is different. The combination of Glauber and Kawasaki dynamics was originally introduced for studying temperature driven non-equilibrium phase transitions. Our focus was the effect of synchronous/asynchronous updating modes on the stationary states in dynamical processes on networks, for which the Ising chain is the simplest case to start a systematic study.

An increase in the dimensionality of the lattice would allow a mean-field description of the transition, since the parity conservation universality class has the critical dimension $d_{c}=2[27,28]$. Our preliminary numerical simulations for this case show qualitatively similar phases as in $d=1$, but with the transition point shifted towards 1 . Our work represents a systematic interpolation between a synchronous and asynchronous updating scheme. The existence of a phase transition sheds some light on the interpretation of stationary states whenever they depend on the updating mode. Above the transition these states may have

Table 1 Critical exponents of the parity conservation (PC) universality class in comparison to the exponents of the synchronous/asynchronous Ising model (SAIM) measured in this paper

\begin{tabular}{llllll}
\hline & $\beta / \nu_{\perp}$ & $\gamma$ & $\delta$ & $v_{\|} / \nu_{\perp}$ & $\eta$ \\
\hline PC & $1 / 2$ & 0 & $2 / 7$ & $7 / 4$ & 0 \\
SAIM & $0.51(1)$ & 0.0 & $0.286(1)$ & $1.746(2)$ & 0.0 \\
\hline
\end{tabular}


lost any remnants of the dynamics below the transition (in our case we end up with an antiferromagnetic state above and a ferromagnetic below the transition). Instead, the stationary states are representative for the dynamics of the updating algorithm itself.

\section{References}

1. Klemm, K., Bornholdt, S.: Pre-print, arXiv:q-bio/0309013 (2003)

2. Greil, F., Drossel, B.: Phys. Rev. Lett. 95, 048701 (2005)

3. Hopfield, J.J.: Proc. Nat. Acad. Sci. USA 79, 2554 (1982)

4. Grondin, R.O., Porod, W., Loeffler, C.M., Ferry, D.K.: Biol. Cybern. 49, 1 (1983)

5. Klemm, K., Bornholdt, S.: Proc. Nat. Acad. Sci. USA 102, 18414 (2005)

6. Klemm, K., Bornholdt, S.: Phys. Rev. E 72, 055101 (2005)

7. Huberman, B.A., Glance, N.S.: Proc. Nat. Acad. Sci. USA 90, 7716 (1993)

8. Block, H.J., Bergersen, B.: Phys. Rev. E 59, 3876 (1999)

9. Arjomandi, E., Fischer, M.J., Lynch, N.A.: J. ACM 30, 449 (1983)

10. Newman, M.E.J., Barkema, G.T.: Monte Carlo Methods in Statistical Physics. Oxford University Press, Oxford (1999)

11. Metropolis, N.A., Rosenbluth, M.N., Rosenbluth, A.H., Teller, E., Teller, J.: J. Chem. Phys. 21, 1087 (1953)

12. Samuelsson, B., Troein, C.: Phys. Rev. Lett. 90, 098701 (2003)

13. Drossel, B. Mihaljev, T., Greil, F.: Phys. Rev. Lett. 94, 088701 (2005)

14. Fontanari, J.F., Köberle, R.: J. Phys. 49, 13 (1988)

15. Bollé, D., Busquets Blanco, J.: Eur. Phys. J. B 47, 281 (2005)

16. Potts, R.B.: Proc. Camb. Phil. Soc. 48, 106 (1952)

17. Bollé, D., Busquets Blanco, J.: Eur. Phys. J. B 42, 397 (2004)

18. Nishimori, H.: TITECH report (1997)

19. Li, F., Long, T., Lu, Y., Ouyang, Q., Tang, C.: Proc. Nat. Acad. Sci. USA 101, 4781 (2004)

20. Menyhárd, N., Ódor, G.: J. Phys. A Math. Gen. 28, 4505 (1995)

21. Ódor, G., Menyhárd, N.: Phys. Rev. E 73, 036130 (2006)

22. Jensen, I.: Phys. Rev. E 50, 3623 (1994)

23. Menyhárd, N., Ódor, G.: J. Phys. A Math. Gen. 29, 7739 (1996)

24. Glauber, R.J.: J. Math. Phys. 4, 294 (1963)

25. Cardy, J., Täuber, U.C.: Phys. Rev. Lett. 77, 4780 (1996)

26. Privman, V.: Nonequilibrium Statistical Mechanics in One Dimension. Cambridge University Press, Cambridge (1997)

27. Hinrichsen, H.: Adv. Phys. 49, 815 (2000)

28. Ódor, G.: Rev. Mod. Phys. 76, 663 (2004)

29. Jensen, I.: J. Phys. A Math. Gen. 26, 3921 (1993)

30. ben-Avraham, D., Leyvraz, F., Redner, S.: Phys. Rev. E 50, 1843 (1990)

31. Zhong, D., ben-Avraham, D.: Phys. Lett. A 209, 333 (1995)

32. Janseen, H.K.: Phys. Rev. Lett. 78, 2890 (1997) 